\title{
Corporate Governance and Bank Performance Assessing the Impact of Corporate Governance on Bank Performance
}

\author{
Article by Olowoyeye, Olusola Emmanuel \\ M.sc Finance, FCA, FCTI, Nigeria \\ E-mail: olusola.olowoyeye@betaplusint.com
}

\begin{abstract}
The objective of this paper is to explore the impact of corporate governance on continuous business operations and Bank performance indices in the Nigerian Banking Industry. To explore the relationship between Corporate Governance and Bank performance, a Pooled Least Squares Regression analysis was run on Eview Statistical Package based on the (2005 - 2009) financial data/report obtained from BGL 2010 Banking Report and the financial reports and accounts of Nigerian Banks. The results of the analysis showed a negative interrelationship between corporate governance and bank performance.

This study finds that there exist a negative interrelationship between corporate governance and bank performance. Hence this portends that the adoption and entrenchment of sound corporate governance and frameworks has significant effect on bank performance.

This paper will increase understanding of the relationship of corporate governance and bank performance.
\end{abstract}

Keywords: Corporate Governance, Bank Performance, Type of ownership.

\section{Introduction}

The primary purpose of the study is to investigate the impact of Corporate Governance on Bank Performance in Nigeria

In order to achieve this major objective, some steps would be adopted such as examining the extent to which Nigerian Banks have incorporate sound corporate governance practices and the impact on their Bank performance indices.

The study seeks to answer the following research questions in order to effectively accomplish the research objective:

1. What are the variables influencing bank performance in Nigeria?

2. What is the relationship between corporate governance variables and bank performance?

3. To what extent does bank liquidity affects the relationship between corporate governance variables and bank performance?

4. To what extent does bank activity mix affects the relationship between corporate governance variables and bank performance?

5. To what extent does bank debt-equity ratio affects the relationship between corporate governance variables and bank performance?

6. To what extent does bank loan-deposit ratio affects the relationship between corporate governance variables and bank performance?

The importance of study starts from the fact that banks play a critical financial intermediation role in the Nigerian Financial System, hence failure of corporate governance principles would greatly impact on bank performance.

This study will be of immense benefit to the Board Members, Managing Directors, various cadres of Management staff in the financial services industry in that it would assist them in adopting the best practices in corporate governance that would enhance their bank's Corporate Performance. 
DOI: $10.21522 /$ TIJMG.2015.03.02.Art022

ISSN: $2520-310 \mathrm{X}$

\section{Scope and limitations}

Corporate Governance and Bank performance are broad concepts that cannot be exhaustively dealt with in a work like this. Hence the focus of this research is to examine the impact of corporate governance on the Bank performance indices of Nigerian the banking industry.

The time period that will be used for the data analysis will be limited to the 5 year financial period (2005 - 2009) of Nigerian Banks based on available financial data.

The aim of this research is to obtain a deeper understanding of how banks can adopt good corporate governance practice to ensure better Bank performance management strategies.

Corporate governance is a field in economics that investigates how to secure efficient management of corporations by the use of incentive mechanisms, such as contracts, organizational designs and legislation. This is often limited to the question of improving financial performance, for example, how the corporate owners can secure that the corporate managers will deliver a competitive rate of return, (Encycogov's n.d.)

15 Weaknesses in Corporate Governance of Banks in Nigerian: (1) Board squabbles, (2)Ineffective Board oversight functions, (3) Fraudulent and self-serving practices among members of the board, management and staff, (4) Overbearing influence of chairman or MD/CEO, (5) Weak internal controls, (6) Non-compliance with laid-down internal controls and operation procedures (7) Ignorance of and noncompliance to Banking rules laws and regulations, (8) Passive shareholders.(9) Poor risk management practices resulting in large quantum of non-performing credits including insider-related credits, (10) Abuses in lending, including lending in excess of single obligor limit, (11) Sit-tight Directors, (12) Succumbing to pressure from other stakeholders, (13) Technical incompetence, poor leadership and administrative ability (14) Inability to plan and respond to changing business circumstances (15) Ineffective management information system.

\section{Effective corporate governance charter}

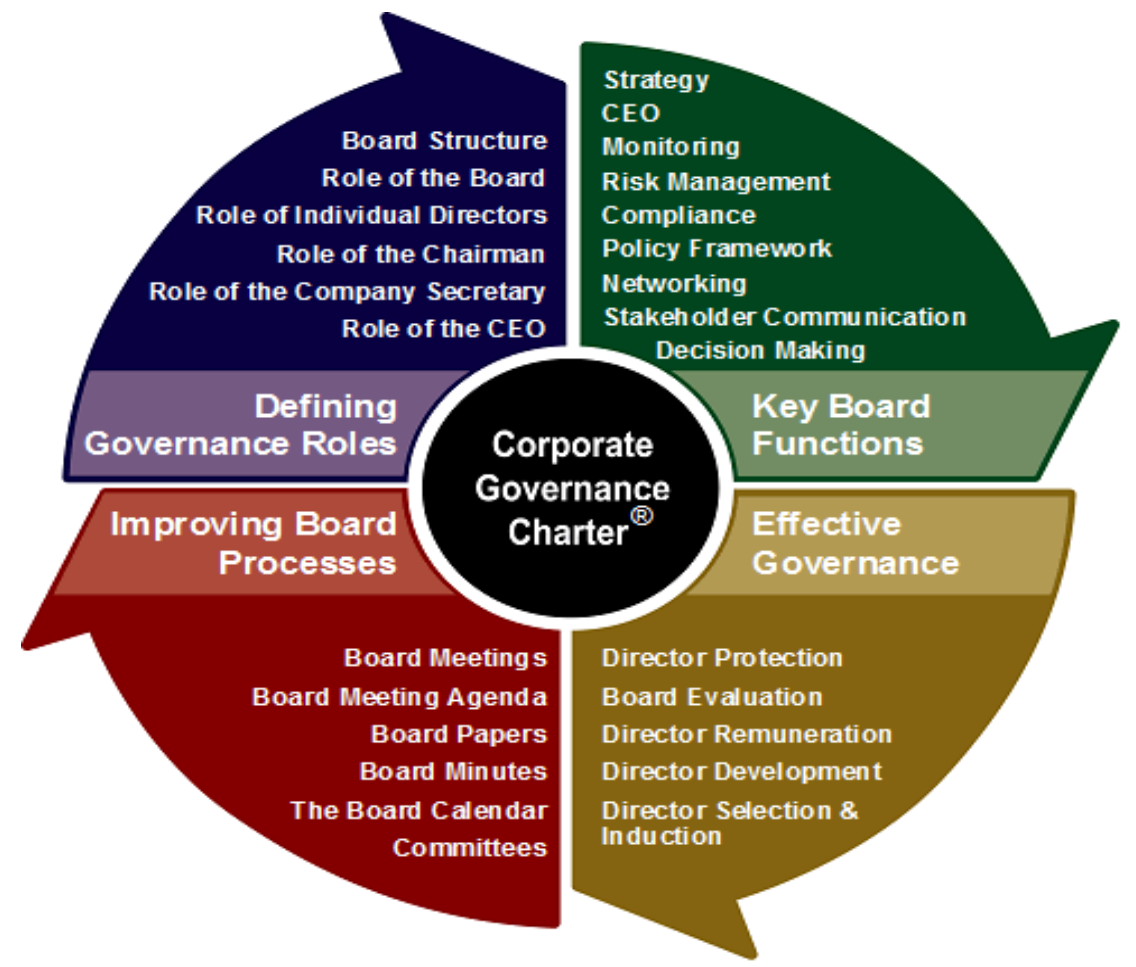

Source: Corporate Governance Charter - Adapted from: Kiel, G.C. and G.J. Nicholson, 2003, Boards that Work: A New Guide for Directors, Sydney: McGraw Hill. 


\section{Bank performance}

Some financial ratios used to evaluate Bank Performance are Return on Average Asset (ROBT), Loan to Loss Ratio, Cost to Income Ratio, Capital Adequacy Ratio (CAR), and Liquidity

The Central Bank of Nigeria (CBN) is the apex regulatory authority of the financial system. It was established by the Central Bank of Nigeria Act of 1958 and commenced operations in July 1959. Among its primary functions, the CBN promotes monetary stability and a sound financial system, and acts as banker and financial adviser to the Federal Government. It is the banker of last resort to licensed banks 24 and approves licences to financial institutions, which include Deposit Money Banks, Primary Mortgage Institutions, Microfinance Banks, Finance companies, Bureaus De Change, and Development Finance Institutions

The work of (Rogers, 2008) indicated that Corporate Governance (Transparency, Trust and Disclosure) predicts $34.5 \%$ of the variance in the general financial performance of Commercial banks in Uganda. The significant contributors to financial performance were openness and reliability.

\section{Statement of hypothesis}

The major arguments of this study are compressed in the following hypothesis:

$\mathrm{HO}$ - There is negative inter-relationship between corporate governance and bank performance.

H1 - Better corporate governance will lead to better bank performance.

\section{Model specification}

Following Flamini, McDonald, and Schumacher (2009) among others, this study adopts a model of bank performance determinants to investigate the relationship between corporate governance and bank performance. Thus, the models to be adopted in this study are as follows:

\section{Model 1}

$$
\begin{aligned}
& \mathrm{ROBT}=\alpha_{0}+\beta_{1} \mathrm{CAP}+\beta_{2} \mathrm{LD}+\beta_{3} \mathrm{LL}+\beta_{4} \mathrm{OWN}+\beta_{5} \mathrm{LIQ}+\beta_{6} \mathrm{ACT}+\beta_{7} \mathrm{SIZE}+\beta_{8} \mathrm{CI} \\
& +\beta_{9} \mathrm{MP}+\beta_{10} \mathrm{DE}+\beta_{12} \mathrm{CTRI}+\text { staff }+\mathrm{MVB}+\varepsilon_{1}
\end{aligned}
$$

\begin{tabular}{|c|c|c|}
\hline ROBT & $=$ & Profitability (Return on Asset) of bank \\
\hline CAP & $=$ & Capital Adequacy Ratio \\
\hline LD & $=$ & Loan to Deposits Ratio \\
\hline LL & $=$ & Loan Loss Provisioning \\
\hline OWN & $=$ & Ownership Structure \\
\hline LIQ & $=$ & LIQ = Liquidity ratio of bank \\
\hline ACT & $=$ & $\mathrm{ACT}=$ Activity Mix \\
\hline SIZE & $=$ & Size of bank \\
\hline $\mathrm{CI}$ & $=$ & Cost Income Ratio \\
\hline MP & $=$ & Market Power of bank \\
\hline $\mathrm{DE}$ & $=$ & Debt-Equity \\
\hline ADIV & $=$ & Quoted Bank \\
\hline DLB & $=$ & Controlling Interest \\
\hline MVB & $=$ & Market to Book value \\
\hline STF & $=$ & Staff Cost \\
\hline QB & $=$ & Quoted Banks \\
\hline CTRI & $=$ & Controlling Interest \\
\hline D1 & $=$ & $=1$ for Private Locally-owned bank, and 0 for others \\
\hline$\alpha$ & $=$ & $=$ Intercept \\
\hline$\square$ & $=$ & $=$ Coefficient of parameters \\
\hline$\square$ & $=$ & $=$ Residual error \\
\hline
\end{tabular}

Where; 
DOI: $10.21522 /$ TIJMG.2015.03.02.Art022

ISSN: $2520-310 \mathrm{X}$

\section{Model 2}

$$
\begin{aligned}
& \mathrm{ROBT}=\alpha 0+\square 1 \mathrm{CAP}+\square 2 \mathrm{LD} \square \square \square 3 \mathrm{LL}+\square 4 \mathrm{OWN}+\square 5 \mathrm{LIQ} \square \square \square 6 \mathrm{ACT}+\square 7 \mathrm{SIZE}+\square 8 \mathrm{CI} \\
& \square \square 9 \mathrm{MP}+\square \text { 10DE } \square \square \square 12 \mathrm{CTRI}+\mathrm{staff}+\mathrm{MVB}+\square 1(3.1)
\end{aligned}
$$

\section{Limitations of the methodology}

The limitations of this study arise from the fact that there is inadequate data needed to embark on a more rigorous study about the Nigerian banks.

Also the regression model adopted was also constrained by limited time series data; hence an unbalanced pooled regression analysis was adopted. The limitations associated with Pooled Least Squares method of Panel Data Regression analysis is worthy of note as well.

The limited scope of time, resources constituted another major constraint in the conduct of this research. The series of changes in the Nigerian Banking Industry in the intervening period constitutes a limitation as well.

Despite these inherent limitations the results obtained largely conforms to the research findings of some other researchers in this and related field of study.

\section{A priori expectation of the relationship}

For the variables investigated in this study it is expected that Corporate Governance will have a negative relationship with Bank Performance.

\section{Analysis of data and interpretation of results}

$$
\begin{aligned}
& \text { ROBT = Profitability (Return on Asset) of bank } \\
& \text { CAP = Capital Adequacy Ratio } \\
& \text { LD = Loan to Deposits Ratio } \\
& \text { LL = Loan Loss Provisioning } \\
& \text { OWN = Ownership Structure } \\
& \text { LIQ = Liquidity ratio of bank } \\
& \text { ACT = Activity Mix } \\
& \text { SIZE = Size of bank } \\
& \text { CI = Cost Income Ratio }
\end{aligned}
$$

\section{Descriptive statistics and analysis}

Table below shows the summary statistics of all the variables used in the study which includes the following : Return on Assets (ROBT), Liquidity ratio (LIQ), Loan loss Provision(LL), Capital adequacy (CAP), Loan-deposit ratio (LD), Activity mix (ACT), Size of the bank (SIZE), Cost-Income Ratio (CI), Market power (MP), Debt-equity ratio (DE), Staff Cost (STAFF), Market to book value equity (MVB), Domestic or Foreign ownership (OWN), Controlling interest of at least $10 \%$ shareholding (CTRL) and Quotation Status (QB).

The descriptive statistics indicates that whilst the mean performance of banks based on ROBT was about 90\% the Cost to Income Ratio (CI) absolute was about $67 \%$ and CAR about 16\%, showing some relationship which is described further in the regression analysis. The negative skewness of the bank 
performance indicators vis-à-vis the positive skewness of the Corporate Governance and variables are indicators of some interrelationships described further in the regression analysis. 


\begin{tabular}{|c|c|c|c|c|c|c|c|c|c|c|c|c|c|}
\hline 竧 & $\stackrel{\sim}{~}$ & तิ & तิ & तి & సి & ते & ती & तิ & तิ & ते & ㅇ| & సిని & तి \\
\hline 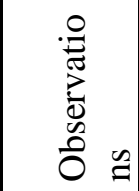 & f & fo & ? & o & fo & fo & 이 & fo & of & fo & fo & fof & fo \\
\hline
\end{tabular}

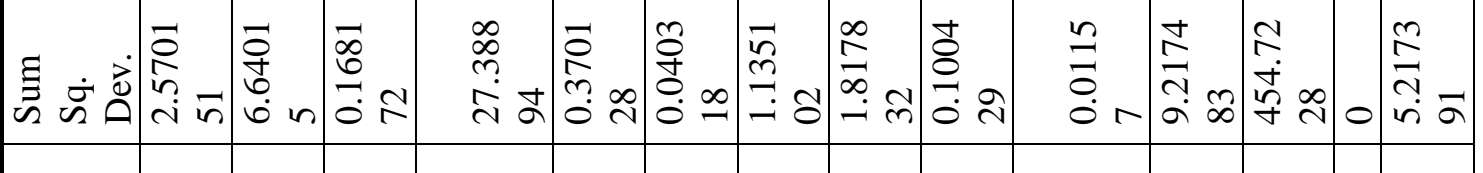

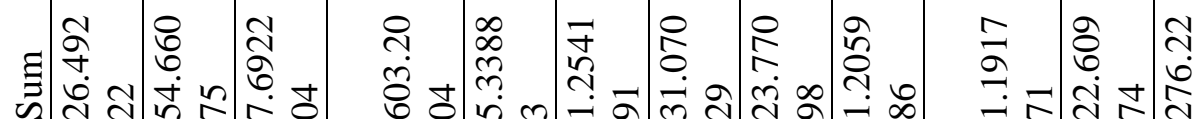

\begin{tabular}{|c|c|c|c|c|c|c|c|c|c|c|c|c|c|}
\hline $\begin{array}{l}:= \\
0 \\
\overline{0} \\
0 \\
0 \\
0\end{array}$ & 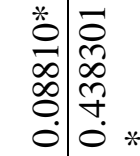 & $\begin{array}{l}0 \\
\infty \\
n \\
n \\
n \\
n \\
0 \\
0\end{array}$ & 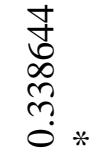 & 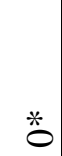 & 苞 & 弟 & 苟 & 苞 & 華 & 苍 & | & $\mathbb{Z}$ & 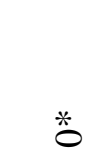 \\
\hline
\end{tabular}

\begin{tabular}{|c|c|c|c|c|c|c|c|c|c|c|c|c|c|c|}
\hline \pm 3 & 2 & $\stackrel{t}{0} *$ & $\tilde{o}_{0}^{m} *$ & $\stackrel{n}{0} *$ & \% & है & ô & है & \% & ث & b & $\stackrel{0}{0} *$ & 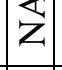 & F \\
\hline 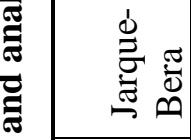 & 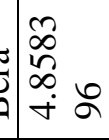 & 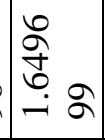 & 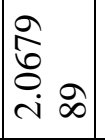 & 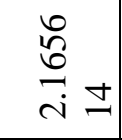 & $\begin{array}{l}\hat{\hat{\alpha}} \\
\infty \\
\stackrel{2}{2} \sigma\end{array}$ & $\mid \begin{array}{l}\infty \\
\infty \\
\dot{0} \\
0 \\
-\infty \\
-\infty\end{array}$ & $\begin{array}{l}\dot{\infty} \\
\dot{\sigma} \\
\stackrel{-}{a} \\
=\end{array}$ & 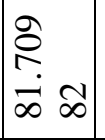 & 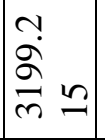 & $\begin{array}{l}\mathbb{N} \\
\infty \\
\infty \\
\sim \\
\infty\end{array}$ & 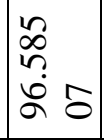 & 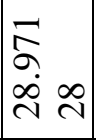 & & 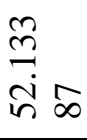 \\
\hline 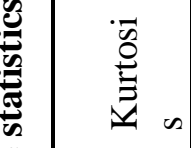 & 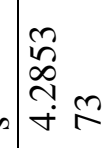 & 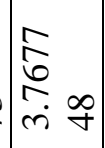 & 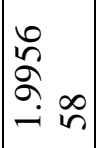 & $\begin{array}{l}\stackrel{+}{0} \\
\stackrel{+}{-} \\
-\end{array}$ & 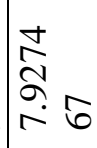 & $\mid \begin{array}{l}2 \\
2 \\
\stackrel{\infty}{0} \infty \\
\infty\end{array}$ & 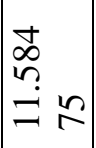 & 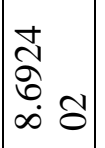 & $\mid \begin{array}{l}\vec{D} \\
\infty \\
\vec{\gamma}=\end{array}$ & $\begin{array}{l}\text { 京 } \\
\infty \\
\dot{ \pm} \\
-\end{array}$ & $\mid \begin{array}{l}\vec{m} \\
\stackrel{\sigma}{\infty} \infty \\
\infty\end{array}$ & 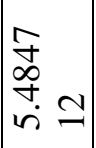 & z) & 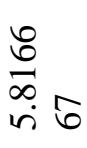 \\
\hline 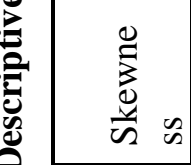 & $\hat{b}$ & 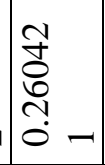 & 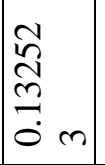 & $\begin{array}{l}\tilde{S} \\
\tilde{0} \\
0 \\
0\end{array}$ & $\begin{array}{l}\infty \\
\tilde{n} \\
\infty \\
0 \\
i\end{array}$ & $\begin{array}{l}\stackrel{n}{f} \\
\\
i \\
i\end{array}$ & $\begin{array}{l}2 \\
\hat{\sigma} \\
\hat{\circ} \\
\hat{n} \\
i\end{array}-1$ & 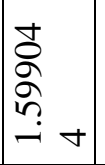 & 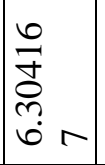 & 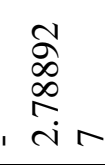 & 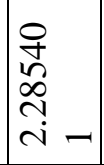 & $\begin{array}{l}m \\
\bar{n} \\
\stackrel{a}{q} \\
-\end{array}$ & & $\begin{array}{l}\stackrel{8}{0} \\
\stackrel{+}{a} \\
\vec{i}-1\end{array}$ \\
\hline$\ddot{~} \tilde{0}$ & $\hat{n}$ & 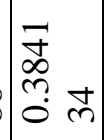 & 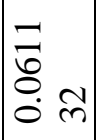 & $\begin{array}{l}\vec{D} \\
\stackrel{\infty}{*} \\
\dot{0}\end{array}$ & 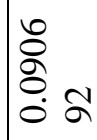 & 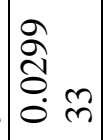 & $\begin{array}{l}\infty \\
\infty \\
n \\
0 \\
0 \\
0\end{array}$ & 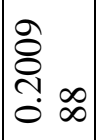 & 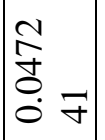 & $\begin{array}{l}\stackrel{8}{\circ} \\
\stackrel{0}{\circ} \mathrm{m}\end{array}$ & 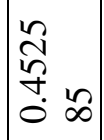 & 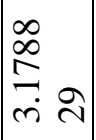 & 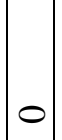 & 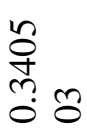 \\
\hline 弯 & $=\mid \begin{array}{l}\infty \\
\infty \\
0 \\
0 \\
0 \\
0\end{array}$ & 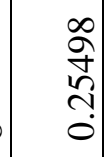 & 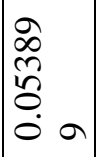 & 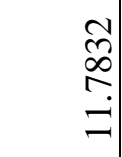 & 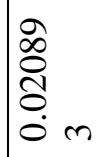 & & 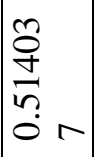 & $\mid \begin{array}{l}\hat{2} \\
\infty \\
\infty \\
0 \\
0 \\
0\end{array}-$ & 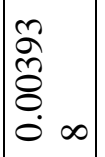 & 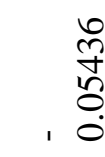 & $\begin{array}{l}0 \\
\vdots \\
0 \\
0 \\
0\end{array}$ & $\begin{array}{l}8 \\
0 \\
o \\
o \\
i \\
i\end{array}$ & - & 0 \\
\hline 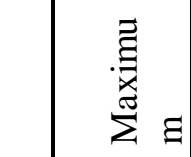 & 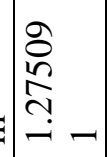 & $\begin{array}{l}\tilde{n} \\
\tilde{c} \\
\tilde{n} \\
\tilde{c} \\
i \\
i\end{array}$ & 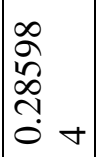 & 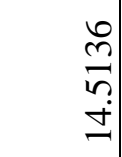 & 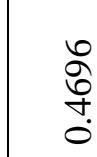 & 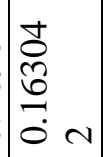 & 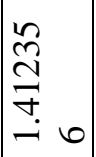 & 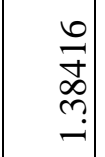 & 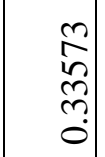 & $\begin{array}{l}\frac{0}{\sigma} \\
\frac{\sigma}{0} \\
\dot{0}\end{array}$ & 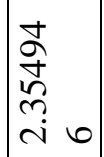 & 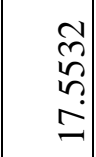 & - & - \\
\hline$\Sigma$ & 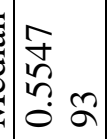 & 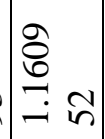 & $\mid \begin{array}{l}\overline{\tilde{b}} \\
\stackrel{2}{0} \\
\dot{0}\end{array}$ & $\begin{array}{l}\stackrel{ \pm}{=} \\
\stackrel{\dot{m}}{=}\end{array}$ & 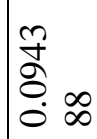 & $\mid \begin{array}{l}\infty \\
\infty \\
0 \\
0 \\
0\end{array}$ & 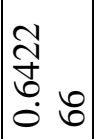 & 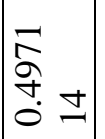 & 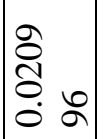 & $\begin{array}{l}\stackrel{0}{\hat{\sigma}} \\
\text { O. } \\
0\end{array}$ & $\begin{array}{l}2 \\
\text { ले } \\
\\
0\end{array}$ & $\mid \begin{array}{l}\tilde{n} \\
\tilde{z} \\
\dot{\delta} \\
i\end{array}$ & 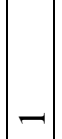 & 0 \\
\hline & 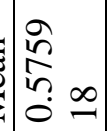 & $\begin{array}{l}\infty \\
\infty \\
\stackrel{\infty}{=} \\
=\end{array}$ & 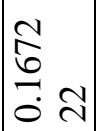 & $\stackrel{m}{=}$ & 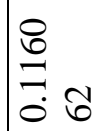 & 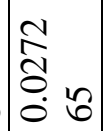 & 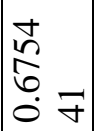 & $\left|\begin{array}{ll}\pi & \\
0 & \\
n & \\
0 & 0\end{array}\right|$ & 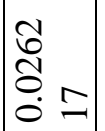 & 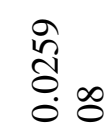 & $\mid \begin{array}{l}n \\
\frac{a}{9} \\
0\end{array}$ & $\begin{array}{l}\infty \\
0 \\
0 \\
0 \\
0 \\
0\end{array}$ & & $\begin{array}{l}\stackrel{t}{0} \\
\stackrel{n}{0} \\
\stackrel{0}{0} m\end{array}$ \\
\hline & -1 & $\hat{Z}$ & ن & & $\Sigma$ & $\exists$ & 已| & $\stackrel{0}{0}$ & 唫 & $\begin{array}{l}\text { to } \\
\stackrel{0}{\simeq} \text {. }\end{array}$ & $\sum_{\Sigma}^{\infty}$ & $\boldsymbol{n}$ & & 。 \\
\hline
\end{tabular}




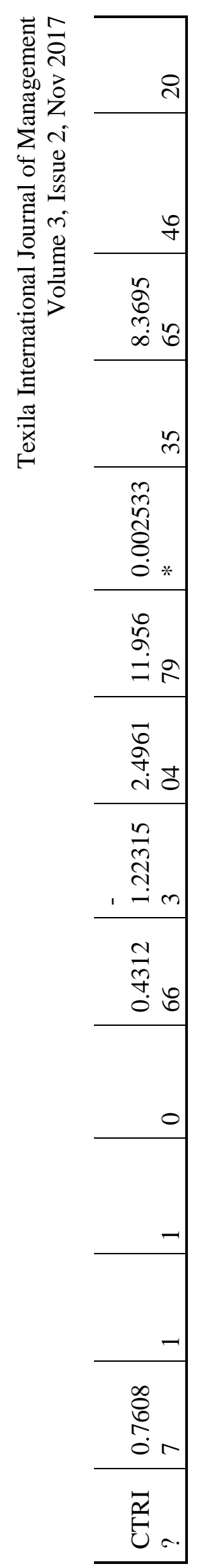


DOI: $10.21522 /$ TIJMG.2015.03.02.Art022

ISSN: $2520-310 \mathrm{X}$

\section{Regression analysis and results}

The summary regression analysis and results for the study as depicted in Tables below is presented and analyzed in this section.

Table 2. Regression results of bank performance model

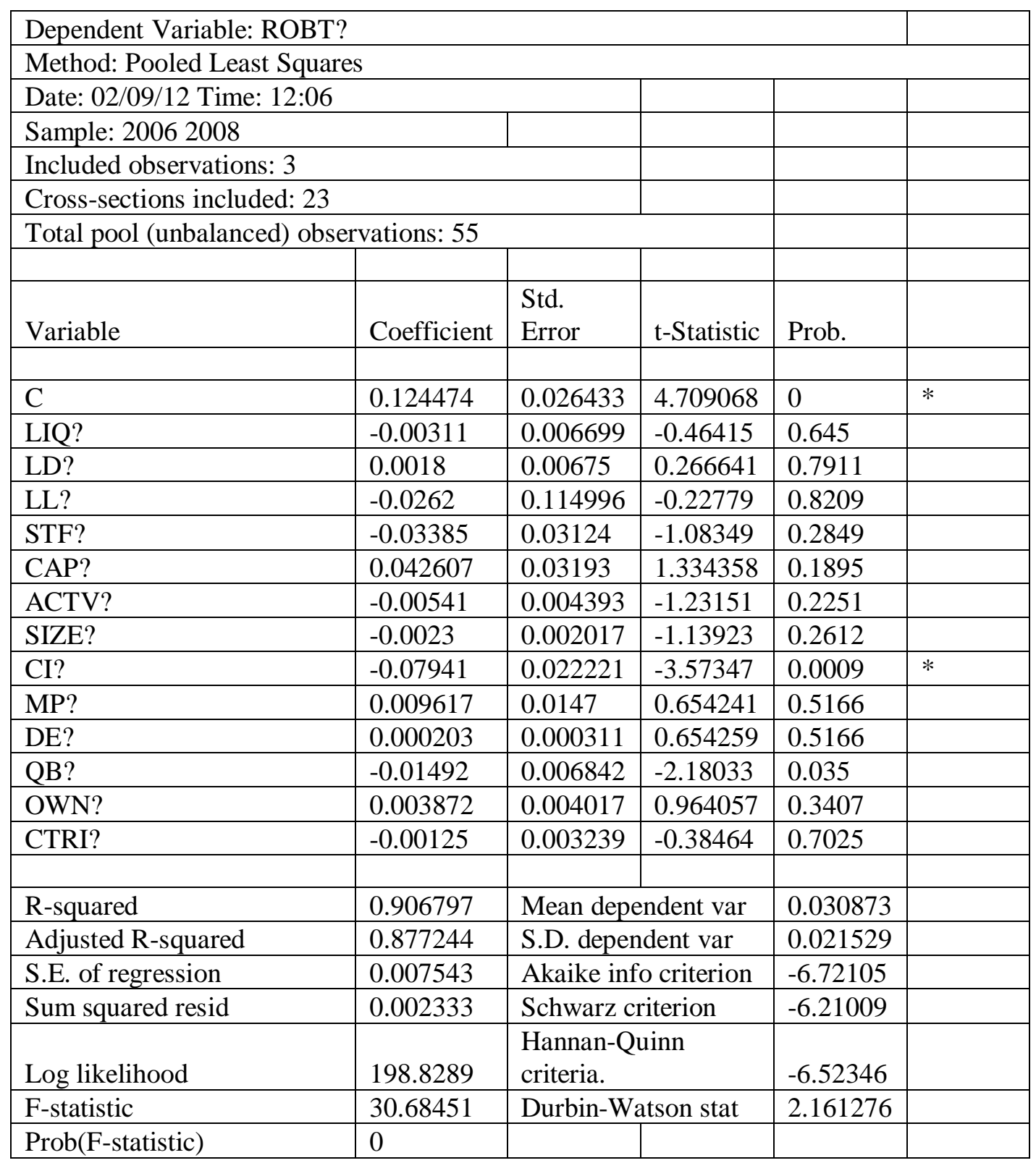

* Significant at the $5 \%$ level

** Significant at the $10 \%$ level

The table shows the results of Model I - estimating the relationship between corporate governance variables and bank performance for all banks. The result in Table 4.1 shows that CI and QB are both negative and significant at the 5\% level while all other variables are insignificant. This appears to show that Cost-income ratio and Quotation status of banks negatively influence bank performance. 
This result also confirms the first hypothesis (H10), which states that there is a negative inter-relationship between bank performance and cost to income ratio.

In the model, $\mathrm{R}$ squared was 0.90 , meaning about $90 \%$ of the variance in ROBT is explained by the model.

Whilst an Adjusted R-squared 0.88 , meaning about $88 \%$ of the variance in ROBT is explained by the model

Durbin-Watson stat of 2.0757 indicates that serial autocorrelation does not exist amongst the variables.

Durbin-Watson should be between 1.5 and 2.5 indicating the values are independent

Hence there is the need to manage these variables in order to attain unto a very higher levels of bank performance.

\section{Summary of findings}

The regression results obtained from this study largely confirms the hypotheses of this study that:

- Better corporate governance and would improve Bank performance measures in respect of banks.

- Better corporate governance is facilitated ensuring that the variables in the corporate governance regression model are well managed by the entity.

Conclusions and recommendations

This study examined the impact of corporate governance on bank performance. The study attempted to answer questions towards determining to what extent the following variables: liquidity, loan loss ratio, and activity mix, staff cost, market power, market-to-book value ratio, capital adequacy, cost-income ratio, ownership, controlling interest and debt-equity ratio a measure of corporate governance and bank performance in the Nigerian banking industry.

Using data over the period of 2005 to 2009 , the study used pooled regression technique to determine the impact of corporate governance on bank performance.

The findings of this study showed that corporate governance has a negative effect on bank performance; sound corporate governance framework would lead to improved Bank performance indices.

The findings of this study further confirm the hypothesis that better corporate governance leads to better performance.

\section{Implication of findings}

The implications of these empirical research findings are significant in nature.

The Banks' board and management should be actively involved in managing all the variables that are both qualitative and quantitative measures of corporate governance and bank performance.

It is a cursor that the regulatory bodies such the Central Bank of Nigeria (CBN), Nigerian Deposit Insurance Corporation (NDIC) should overhaul their bank supervisory and regulatory framework with the objectives of preventing corporate banking failures that are a resultant effect of corporate governance and banks performance;

Various stakeholders in the banking industry - depositors, customers, shareholders, etc, should be involved in various degrees and levels to ensure that unethical and unwholesome practices that are risky in nature are curbed before they become a contagion on the entire system and resulting in grave losses;

\section{Recommendations}

- The regulatory bodies in the Nigerian Financial System should constantly review the level of banks compliance with Corporate Governance Codes;

- In similar vein various measures should be adopted by regulatory bodies to ensure compliance by banks as regards corporate governance codes;

- The banks that is the focus of this study should review their present corporate governance framework and their indices of Bank performance as the starting point; 
DOI: $10.21522 /$ TIJMG.2015.03.02.Art022

ISSN: $2520-310 \mathrm{X}$

- Beyond adopting the letter of corporate governance codes, the board and management of the banks should institute a culture wherein sound governance measures is seen adopted as a corporate way of life;

- The board and management of banks should review the various indices of Bank performance in the light of corporate governance frameworks, since this impact on banks' performance, survival in the corporate landscape.

Corporate governance can be a powerful lever for change throughout the banking industry. Archimedes, the great mathematician once says that, given firm place to standard with a lever he could move the earth. If the same lever of corporate governance is pulled throughout the nation's economy, we can expect some movement to change organizational cultures throughout the Banking sector.

The leading institution in all sectors of the national economy should embrace corporate governance. Stock Exchange and Capital Markets authority can influence the listed purpose especially through listing requirements and annual reports. The central bank can enforce corporate governance on licensed commercial bank that in turn would similarly do so unto her corporate customers and state enterprises, thereby stopping fiscal herm phage and encouragement there are real contributions to GDP, and in the private sector to a dynamic enterprise, culture and increased performance and profitability of private companies leading both increase export and increase rate of GDP growth

\section{Conclusions}

This study examined the impact of corporate governance on bank performance in the Nigerian banking industry. Various recommendations were proffered to regulatory bodies, board members and management of banks such that if adopted would ensure survival, stability, and sustained profitability of the Nigerian Banking Industry in the national and global corporate landscape.

\section{Reference}

[1]. Available at: http://www.crrconference.org/downloads/2006rogers.pdf [Accessed 17 June 2010].

[2]. Available at: http://www.cenbank.org/OUT/PUBLICATIONS/BSD/2006/CORPGOV-POSTCONSO.PDF [Accessed 18 June 2010].

[3]. Adams, R. B. \& Mehran, H., 2008. Corporate Performance, Board Structure, and Their Detinants in the Banking Industry. Federal Reserve Bank of New York Staff Reports, pp. 10-24.

[4]. Blaauw, A., 2009. From to Governance Risk and Compliance. Vanguard Newspaper, 8 November 2009.

[5]. Buehler, K., Freeman, A. \& Hulme, R., 2008. The New Arsenal of Risk Management. Harvard Business Review, 1 September 2008.

[6]. Berger, A. N. et al., June 2005. Corporate Governance and Bank Performance: A Joint Analysis of the Static, Selection, and Dynamic Effects Domestic, Foreign, and State Ownership. pp. 9-26.

[7]. Biswas, P. K. \& Bhuiyan, H. U., 2008. Corporate Governance and Firm Performance: Theory and Evidence from Literature. University of Western Australia. [Online] Available at: http://ssrn.com/abstract=1257617[Accessed 18 June 2010].

[8]. Central Bank of Nigeria (CBN), 2006. Code of Corporate Governance for Banks in Nigerian Post Consolidation. [Online] Baber, W. R. \& Liang, L., 2008. Associations between Internal and External Corporate Governance Characteristics and the Consequences of Regulating Governance Practices. [Online] Available at SSRN: http://ssrn.com/abstract=1146922[Accessed 18 June 2010].

[9]. Hampton, J. J., 2009. Fundamentals of: how top companies assess risk, manage exposure, and seize opportunity. 1st ed. New York: American Management Association.

[10]. Kajola, S. O., 2008. Corporate Governance and Firm Performance: The Case of Nigerian Listed Firms. European Journal of Economics, Finance and Administrative Sciences, ISSN 1450-2887(14 (2008)), pp. 22-25.

[11]. Kirkpatrick, G., 2010. Corporate Governance in the Wake of the Financial Crisis, New York and Geneva: United Nations. 
[12]. KPMG Australia, 2003. : An Emerging Model for Building Shareholder Value. [Online] Available at: http://www.kpmg.com.au/aci/docs/ent-risk-mgt.pdf[Accessed 10 June 2010].

[13]. Rogers, M., 2008. Corporate Governance and Financial Performance of Selected Commercial Banks in Uganda. [Online]

[14]. "Organization of Economic Cooperation and Development (OECD)." April 1999

[15]. The Institute of Directors in Southern Africa, 2009. King Code of Governance for South Africa. [Online] Available at: http://www.ecgi.org/codes/documents/king3.pdf[Accessed 17 June 2010].

[16]. Thomson, D. \& Jain, A., First Quarter 2006. Corporate Governance Failure And Its Impact On National Australia Bank's Performance. Journal of Business Case Studies - First Quarter 2006, 2(1), pp. 41-52.

[17]. The Institute of Directors in Southern Africa. "King Code of Governance for South Africa." 2009. http://www.ecgi.org/codes/documents/king3.pdf (accessed June 17, 2010).

[18]. Thomson, Dianne, and Ameeta Jain. "Corporate Governance Failure And Its Impact On National Australia Bank’s Performance." Journal of Business Case Studies - First Quarter 2006 (Deakin University, Australia) 2, no. 1 (2006): 41-52.

[19]. Tandelilin, E., Kaaro, H., Mahadwartha, P. K. \& Supriyatna, 2007. Corporate Governance, Risk Management, and Bank Performance: Does Type of Ownership Matter? [Online] Available at: http://www.eadn.org/eduardus.pdf [Accessed 17 June 2010].

[20]. The Financial Reporting Council, 2010. The UK Corporate Governance Code. UK. [Online] Available at: http://www.frc.org.uk/documents/pagemanager/corporate_governance/uk\%20corp $\% 20$ gov\%20code $\% 20$ june $\% 2$ 02010.pdf [Accessed 17 July 2010].

[21]. Uchendu, O.A. "Banking Sector Reforms and Bank Consolidation: The Malaysian Experience. Bullion, 29 (2.)." (2005).

[22]. Viverita, 2008. The Effect of Mergers on Bank Performance: Evidence from Bank Consolidation Policy in Indonesia. International Review of Business Research Papers, 2 July 2008, 4(4), pp. 372-377.

[23]. Vishny, Shleifer and. The Journal of Finance, 1997: 737.

[24]. Viverita. "The Effect of Mergers on Bank Performance: Evidence from Bank Consolidation Policy in Indonesia." International Review of Business Research Papers 4, no. 4 (July 2008 2008): 372-377.

[25]. Wentzel, Webber. "King III Report on Governance launched." September 2, 2009. 